\section{$\underset{\substack{\text { hommes } \\ \text { \& migrations }}}{ }$}

\section{Hommes \& migrations}

Revue française de référence sur les dynamiques

migratoires

1277 | 2009

France - Allemagne

\title{
La représentation politique des migrants en Allemagne
}

\section{Andreas M. Wüst et Dominic Heinz}

Traducteur : Fritz Wittek-Kaïm

\section{(2) OpenEdition}

\section{Journals}

\section{Édition électronique}

URL : http://journals.openedition.org/hommesmigrations/157

DOI : 10.4000/hommesmigrations. 157

ISSN : 2262-3353

Éditeur

Musée national de l'histoire de l'immigration

Édition imprimée

Date de publication : 1 janvier 2009

Pagination : $36-46$

ISSN : 1142-852X

Référence électronique

Andreas M. Wüst et Dominic Heinz, "La représentation politique des migrants en Allemagne ", Hommes \& migrations [En ligne], 1277 | 2009, mis en ligne le 29 mai 2013, consulté le 02 mai 2019. URL : http://journals.openedition.org/hommesmigrations/157; DOI : 10.4000/ hommesmigrations. 157 


\section{La représentation politique des migrants en Allemagne ${ }^{(1)}$}

Par Andreas M. Wüst, et Dominic Heinz, chargés de cours en sciences politiques, chercheurs au centre de Mannheim pour la recherche en sciences sociales européennes

Graphique 1 : Affiliations de tous les élus allemands et des élus issus de limmigration aux commissions du Bundestag, des parlements régionaux et européen cumulés (octobre 2007, en pourcentage de toutes les affiliations).

Depuis l'après-guerre, la société allemande a accueilli de nombreux migrants. Le cadre légal de l'immigration a changé et, malgré un déficit linguistique, les immigrés accomplissent aujourd'hui leur intégration politique au sein des différents parlements. Même si le pourcentage d'élus

issus de l'immigration reste moindre que celui des immigrés dans la population, ces élus symbolisent une société devenue multiethnique et profitent de partis de gauche plus ouverts que ceux de droite. Leur activité d'élu se révèle plus intense que celle de leurs confrères et elle s'oriente vers les questions relatives à l'immigration sans pour autant s'y limiter. 
Dans la deuxième moitié $\mathrm{du} \mathrm{Xx}^{\mathrm{e}}$ siècle, l'Allemagne est devenue un pays multiethnique à la faveur de l'immigration de travailleurs, des Aussiedler ${ }^{(2)}$ venant du bloc soviétique et de nombreux refugiés. Bien que ce fait ne soit toujours pas accepté par tous les composants de la société allemande, il fait aujourd'hui l'objet d'un large consensus dans la classe politique. La CDU en particulier s'est employée depuis la fin des années 1980 à réduire l'écart entre la réalité sociale et ses positions sur l'immigration. Cette dernière n'est plus un sujet tabou, et le débat au sein des instances du parti ne se limite plus à la seule revendication de l'assimilation des étrangers. Bien qu'on puisse douter que l'Allemagne ait opéré un changement de paradigme fondamental et définitif en matière de politique d'immigration, on ne peut nier que la réforme du droit de la citoyenneté de 1999 ait constitué un changement significatif du cadre légal de l'immigration en Allemagne. ${ }^{(3)}$ Peu après, la loi relative à l'immigration de 2004 n'a pas seulement autorisé le séjour temporaire, mais elle a aussi institué un droit à l'établissement durable et donc, in fine, à l'immigration de travailleurs hautement qualifiés. Aussi petit soit-il, ce pas vers une ouverture plus large du pays à des immigrants potentiels reste notable, quand bien même, pour l'instant, il se limiterait à la catégorie étroitement circonscrite des travailleurs hautement qualifiés.

\section{Le déficit linguistique des immigrés}

La reconnaissance de la réalité de l'immigration n'a pas seulement provoqué les réformes du droit d'entrée sur le territoire et du droit d'établissement et de naturalisation ; elle a aussi suscité des changements en matière de politiques d'intégration. À cet égard, les résultats des élèves issus de l'immigration dans la première enquête PISA (2000) ont eu des effets mobilisateurs très importants. Une scolarisation déficitaire entraîne de graves handicaps lors de l'entrée sur le marché du travail. Chez les élèves issus de l'immigration, les trop fréquentes lacunes des connaissances en allemand sont la cause la plus importante des retards scolaires. De ce point de vue, l'intégration socio-économique des immigrés et de leurs descendants dépend en tout premier lieu de leurs compétences linguistiques. ${ }^{(4)}$ Si ce résultat de la recherche en sciences sociales n'est pas contesté, on peut néanmoins douter que seules les compétences linguistiques des immigrés, et conséquemment, leur assimilation structurelle, suffisent à garantir leur intégration complète et celle de leurs descendants. Existe-t-il réellement un effet d'entraînement de l'assimilation structurelle des immigrés au sein de la société allemande qui ne s'arrêterait pas devant les institutions politiques que sont les partis et les parlements? 


\section{Intégration politique et représentation parlementaire}

Plusieurs raisons, parmi lesquelles l'hypothèse d'une volonté de démarcation ethnique et culturelle de la majorité dominante, font penser que de tels effets de spill-over (renversement) vers la politique et les parlements ou bien n'existent pas du tout, ou bien ne se produisent que de manière ponctuelle et avec des retards considérables.

On ne peut pas exclure une instrumentalisation de l'origine, que ce soit à des fins carriéristes de la part de candidats issus de l'immigration, ou de la part des partis à des fins de mobilisation électorale.
Aussi, on peut douter que les partis politiques offrent les mêmes perspectives aux immigrés qu'aux ressortissants allemands. ${ }^{(5)}$ Les tenants de positions conservatrices en matière de politiques sociétales, qui - dans l'éventualité de conflits culturels, à ne pas confondre avec des aspects structurels tels que la compétence linguistique ou la durée de la scolarité - se situeraient du côté de la culture dominante, hésiteront avant de s'ouvrir à des personnes ou à des catégories de personnes qui n'appartiennent pas clairement à la culture dominante. ${ }^{(6)}$ Bien qu'il faille se garder d'établir des parallélismes entre des développements au sein même de la majorité d'un côté, et entre majorité dominante et minorités de l'autre, l'exemple de l'intégration des femmes - globalement déficitaire quoique très variable d'un parti politique à l'autre - démontre l'existence de mécanismes de mise à l'écart et d'exclusion. En tout état de cause, l'hypothèse d'une évolution automatique de l'assimilation structurelle vers la représentation politique semble très peu probable.

Cela dit, l'idée d'une intégration séquentielle (allant de l'intégration cognitive jusqu'à l'identification, en passant par les étapes d'intégration structurelle et sociale), tout comme celle d'un spill-over de la dimension structurelle vers la dimension politique, ne correspond qu'à l'une des perspectives possibles sur l'intégration des immigrés et de leurs descendants. ${ }^{(7)}$ Si à l'inverse, on part de l'intégration politique elle-même, on peut supposer que la présence dans les parlements d'élus issus de l'immigration génère elle-même des effets politiques ${ }^{(8)}$, tout en étant l'aboutissement de parcours d'intégration personnelle réussis. Du côté de la majorité dominante, ces réussites peuvent avoir un effet d'ordre symbolique et favoriser la perception du caractère multiethnique de la société. Du côté des minorités, l'identification avec le système politique du pays d'immigration ${ }^{(9)}$ - voire les satisfactions ressenties par des réussites emblématiques - peut avoir des effets 
concrets sur les politiques poursuivies, à commencer par un regain d'importance des sujets liés à l'immigration, en allant jusqu'à une plus grande réceptivité envers les aspirations et les revendications catégorielles. ${ }^{(10)}$ À l'inverse, on ne peut pas exclure une instrumentalisation de l'origine, que ce soit à des fins carriéristes de la part de candidats issus de l'immigration, ou de la part des partis à des fins de mobilisation électorale.

Quoi qu'il en soit, l'élection de parlementaires issus de l'immigration donne du grain à moudre à la recherche empirique sur la représentation politique. Des aspects clés de cette thématique seront approfondis dans le projet de recherche international et comparatif "Migranten als politische Akteure" (Des migrants acteurs politiques) conduit avec le soutien financier de la fondation Volkswagen. Le texte qui suit en présente les premiers résultats relatifs aux seuls parlements allemands. Ajoutons qu'au moment de la rédaction, la collecte de données concernant le Bundestag était déjà relativement avancée, alors que la collecte de données sur les parlements des Länder en était encore à ses débuts.

\section{Combien d'élus issus de l'immigration?}

Les informations que nous avons pu recenser sur les députés du Bundestag depuis 1990 montrent des évolutions pleinement compréhensibles, à condition de les resituer dans l'histoire de l'immigration en Allemagne fédérale. D'abord, on constatera que plus on s'éloigne de la Seconde Guerre mondiale et des flux migratoires qui en ont résulté, plus le nombre de députés (et de candidats) nés en dehors de l'Allemagne décroît. Encore en 1990, on comptait quatre-vingt-un députés nés en dehors de l'Allemagne (soit 12 \% du total), parmi lesquels soixante-douze Heimatvertriebene $e^{(11)}$ et huit députés nés Allemands à l'étranger. Dans le douzième Bundestag (1990-94), la députée sorabe ${ }^{(12)}$ Angela Stachowa, d'origine tchèque, était le seul membre du Bundestag issu de l'immigration extra-allemande.

Après 1990, le nombre d'Heimatvertriebene au Bundestag baisse constamment, jusqu'à ce qu'en 2005 il n'y en ait plus qu'un seul. En revanche, il y avait douze députés issus de l'immigration extra-allemande - la majorité d'entre eux étant issue de la première génération contrairement à ce qu'on aurait pu supposer. Dans les parlements régionaux et européen, les résultats ne sont pas très différents. Sur un total de trente-sept élus régionaux issus de l'immigration, 54 \% ont eux-mêmes migré vers l'Allemagne, et, parmi les douze membres allemands du Parlement européen qui sont issus de l'immigration, sept sont dans ce cas. De ce point de vue, les différences entre partis sont insignifiantes. 
Au Bundestag, cinq de ses membres sont d'origine turque, quatre appartiennent à la première génération, une seule à la deuxième, auxquels s'ajoutent trois immigrés de première génération originaires de la Pologne, de la Croatie et de l'Iran. Trois députés appartiennent à la deuxième génération, dont l'une est d'origine iranienne, les deux autres étant d'origine indienne. On notera par ailleurs qu'à la différence des parlements régionaux et européen, le Bundestag ne compte pas de députés issus des immigrations italienne, grecque ou espagnole. Dans les parlements régionaux, on compte quatre élus d'origine italienne et un d'origine grecque. Au Parlement européen, un député allemand est d'origine grecque. Au niveau communal (les vingt-cinq plus grandes villes, y compris les arrondissements berlinois), nous avons recensé vingt-trois élus d'origine grecque, sept d'origine italienne et deux d'origine espagnole.

\section{Appartenance partisane et distribution des sièges}

La majorité des élus issus de l'immigration appartiennent à un parti de gauche. Les Verts, suivi du Linkspartei ${ }^{(13)}$, affichent les proportions les plus élevées. En nombre absolu, le SPD compte à peu près autant d'élus issus de l'immigration que les Verts et le Linkspartei réunis. Dans les groupes parlementaires de droite - exception faite du Parlement européen - on rencontre beaucoup moins d'élus issus de l'immigration, quand bien même, au niveau communal, les écarts avec les élus issus de partis de gauche s'amenuiseraient davantage.

De manière générale, la proportion d'élus issus de l'immigration dans les parlements allemands reste très en deçà de la proportion de la population immigrée par rapport à la population totale (environ $19 \%$ ), et même de la proportion des immigrés naturalisés (environ $10 \%$ des immigrés). Cependant, ce phénomène varie à la fois selon les partis et selon le niveau des parlements. Nos données relatives au niveau communal, limitées aux grandes villes, n'autorisent pas à des conclusions de portée générale. En revanche, pour le Parlement européen, nous avons trouvé un nombre plus élevé d'élus issus de l'immigration qu'aux niveaux national ou régional. Ce constat peut s'expliquer par trois raisons : (1) le droit de vote actif et passif pour les citoyens européens ; (2) le fait que pour les partis politiques les élections européennes sont moins importantes ${ }^{(14)}$, et que par conséquent, ces partis seraient moins réticents à investir des candidats issus de l'immigration ; (3) les enjeux politiques au niveau européen, caractérisés par une plus forte dimension transnationale, ne sont pas les mêmes qu'aux niveaux national ou régional.

La composition du groupe des députés européens confirme la première hypo- 
thèse : huit des douze députés européens issus de l'immigration élus en Allemagne sont originaires d'un pays membre de l'Union européenne, trois sont d'origine turque et le douzième est d'origine égyptienne. La situation est différente au Bundestag et dans les parlements régionaux, les élus originaires d'un autre pays de l'Union européenne y constituent une minorité parmi les élus issus de l'immigration. Nous allons examiner la pertinence de la deuxième hypothèse dans le cadre de nos recherches ultérieures, tandis que la troisième sera examinée plus bas.

Les différences entre les partis peuvent aussi être considérées comme autant d'indicateurs des différentiels de perspectives de sièges que les partis allemands proposent aux immigrants et à leurs descendants ; mais un recensement systématique des candidatures, combiné à une analyse de leurs chances de réussite (selon qu'il s'agisse de scrutins majoritaires ou proportionnels ${ }^{(15)}$ ), fournirait un indice plus probant. Il n'empêche que la distribution selon les partis montre que les partis de gauche sont plus ouverts aux personnes issues de l'immigration que ceux de droite. ${ }^{(16)}$ C'est particulièrement vrai pour les élus d'origine turque, qui sont très peu représentés au sein de la CDU (environ $6 \%$ du nombre total des élus issus de l'immigration), et seulement au niveau communal ; en revanche, ils sont mieux représentés au sein du SPD (environ 30 \%), des Verts (environ 42 \%) et du Linkspartei (environ $85 \%$ ), où ils sont proportionnellement les plus nombreux. ${ }^{(17)}$

\section{Prionités et domaines d'activité politique}

La présence au parlement d'élus issus de l'immigration peut, à elle seule, avoir des effets intégrateurs positifs ${ }^{(18)}$, et, pour ce qui est des partis de gauche, il n'y a pas de doute qu'elle a aussi (quoique pas seulement) un fort aspect symbolique. Ainsi, ce n'est pas un hasard si Les Verts ont fait élire Cem Özdemir à la fonction de Schriftführer ${ }^{(19)}$ au Bundestag. Cette fonction étant dotée d'une grande visibilité pour les parlementaires eux-mêmes, pour les médias et pour le public; la seule présence de Cem Özdemir incarne les idées multiculturelles de son parti, même lorsqu'il ne prend pas la parole. ${ }^{(20)}$ L'élection des Schriftführer Juratovic (SGP), Keskin (La Gauche) et Winkler (Les Verts) semble indiquer que cette stratégie a trouvé des imitateurs. ${ }^{(21)}$ Cependant, la seule présence symbolique d'un député n'a pas d'impact sur les politiques menées. Pour en savoir plus sur d'éventuels effets de fond de la présence d'élus issus de l'immigration, il est nécessaire de recenser leurs priorités et leurs domaines d'activité politique.

Cela peut se faire en analysant les informations fournies par les élus eux-mêmes, par exemple sur leurs pages Internet personnelles, où ils peuvent indiquer - sans 
que pour autant tous le fassent - de quels sujets ils s'occupent en priorité au parlement. Une autre méthode, pour élucider leurs sujets de prédilection ainsi que leur influence éventuelle, consiste à analyser leurs activités dans les commissions parlementaires. Il s'agit de vérifier la thèse selon laquelle des élus issus de l'immigration s'occuperaient plus particulièrement de sujets d'immigration, et qu'on les rencontrerait plus souvent dans des commissions traitant plus ou moins directement de sujets en relation avec l'immigration.

Un premier regard rapide sur les députés issus de l'immigration donne effectivement le sentiment qu'ils s'occupent beaucoup - plus souvent en tout cas que d'autres élusde sujets en rapport avec l'immigration. Afin de tester cette impression, nous proposons de

les députés issus de l'immigration siègent proportionnellement plus dans des commissions dont les activités sont en rapport avec l'immigration. regrouper les commissions permanentes du Bundestag et des parlements régionaux et européen sous cet aspect. Dans beaucoup de domaines politiques (éducation et formation, santé...), cela ne pose pas de grands problèmes. En revanche, d'autres commissions (culture, science, média...) s'y prêtent moins facilement, et nous avons regroupé certaines d'entre elles dans une seule et même catégorie. De plus, certaines commissions (défense...) n'existent qu'au niveau du Bundestag, d'autres encore (pêche...) dans quelques parlements seulement, ce qui fait que la comparaison des commissions peut produire une image légèrement biaisée. C'est pourquoi nous avons, pour tous les élus - sans distinction d'origine - de ces trois niveaux de parlements, recensé les commissions dont ils sont membres de plein droit (et non suppléants), afin de pouvoir comparer ces données avec celles concernant les seuls élus issus de l'immigration; de cette manière, nous avons pu identifier les commissions où ces derniers sont surreprésentés ou sous-représentés.

Notons d'abord que dans les parlements analysés, les députés siègent en moyenne dans une seule commission, alors que pour les députés issus de l'immigration cette moyenne monte à $1,3{ }^{(22)}$ S'ils siègent dans plus de commissions que leurs confrères, qui ne sont pas issus de l'immigration, cela semble indiquer une activité politique plus intense et (du moins sur le plan formel) un haut niveau d'intégration dans les groupes parlementaires. Le graphique 1 page 36 montre que les députés issus de l'immigration siègent proportionnellement plus dans des commissions dont les activités sont en rapport avec l'immigration. C'est seulement dans les commissions des affaires sociales que l'on trouve les mêmes proportions; dans les commissions des affaires culturelles, elles sont inférieures. Dans les commissions 
des pétitions, on trouve des proportions particulièrement élevées d'élus issus de l'immigration, ce qui semble montrer que ces derniers acceptent de jouer un rôle de médiateur entre la population et les parlements. Enfin, les commissions des affaires extérieures ou européennes comptent beaucoup d'élus issus de l'immigration, ce qui confirme qu'ils s'intéressent particulièrement à des sujets transnationaux et occupent des fonctions en conséquence.

Dans tous les parlements, les commissions moins directement concernées par des sujets relatifs à l'immigration (en particulier la défense, mais aussi l'agriculture et l'environnement) comptent peu d'élus issus de l'immigration. Seules les commissions des affaires économiques - par ailleurs très hétérogènes - font exception à cet égard. Globalement, on peut dire que les élus issus de l'immigration sont davantage présents dans des commissions susceptibles de traiter de sujets en rapport avec l'immigration. Cependant, si on analyse la situation individuelle des élus issus de l'immigration et leur participation aux commissions, on constatera que leur activité parlementaire ne se limite que rarement à des questions relatives à l'immigration. Le fait de s'occuper de ces questions n'empêche pas la majorité d'entre eux de travailler aussi sur d'autres questions. Quelques-uns seulement se consacrent exclusivement aux questions ayant un rapport avec l'immigration, tout comme à l'inverse très peu d'entre eux semblent vouloir éviter ce type de questions.

Le graphique 2 page 44 montre qu'en analysant l'appartenance aux commissions parlementaires selon les groupes politiques, on trouve des différences semblables, bien que moins prononcées que celles entre les groupes politiques eux-mêmes. Les élus issus de l'immigration de gauche et de droite siègent majoritairement dans les commissions traitant de sujets en relation avec l'immigration, la participation des élus de gauche n'y étant que très peu supérieure à celle de leurs confrères de droite. Pour ce qui est des commissions que nous avons regroupées pour en faire une catégorie thématique, on notera que les élus de droite siègent plutôt dans les commissions des affaires économiques, moins directement concernées par les questions d'immigration, mais aussi dans les commissions des affaires sociales, suivies par les commissions juridiques et de l'éducation, plus directement intéressées. Les élus de gauche sont fortement représentés dans les commissions des affaires étrangères ou européennes, suivies par les commissions des pétitions et des affaires intérieures. Parmi les sujets plus directement importants pour l'immigration, les affaires sociales ainsi que l'éducation et la formation retiennent moins leur intérêt que les affaires internationales, la médiation entre les citoyens et les autorités publiques, ainsi que les intérêts des immigrés.

À titre provisoire, on peut conclure que les parlementaires issus de l'immigration s'occupent prioritairement, quelle que soit leur obédience politique, de sujets en 
Graphique 2 : appartenance des élus issus de l'immigration aux commissions du Bundestag, des parlements réglonaux et européen cumulés, par partis politiques (octobre 2007, en pourcentage de toutes les affiliations).

relation plus ou moins directe avec les problématiques migratoires, dans des commissions différentes, selon des modalités différentes et très probablement avec des objectifs différents. En revanche, des efforts de recherche ultérieurs seront nécessaires pour répondre à la question de savoir en quoi consistent précisément ces différences, et si, en matière de représentation des personnes issues de l'immigration, il existe de réelles différences entre les partis.

\section{Des migrants devenus acteurs politiques}

Les processus migratoires ont une dimension politique propre. Le pronostic établi il y a vingt-sept ans par Mark J. Miller ${ }^{(23)}$ ne se réalise que lentement; en Allemagne aussi, les immigrés et leurs descendants sont devenus des acteurs politiques. Qu'ils soient électeurs, candidats ou parlementaires, jusqu'à l'heure actuelle, leurs préférences politiques vont assez clairement vers les partis de gauche. ${ }^{(2)}$ Cela peut s'expliquer par le fait que pour le SPD et Les Verts les politiques de justice sociale et d'intégration ne se sont jamais arrêtées devant des frontières ethniques ou culturelles. Les partis de droite ont manifestement plus de mal. S'ils comptent aujourd'hui - dans les villes en particulier - quelques élus issus de l'immigration (de première, deuxième ou troisième génération) dans leurs rangs, il n'y a guère d'élus d'origine turque en leur sein. 
À observer les élus issus de l'immigration au Bundestag, dans les parlements des Länder et au Parlement européen, on constatera indépendamment de leur affiliation politique, une activité plus forte sur des sujets en relation étroite avec les problématiques migratoires. Ainsi, ces activités spécifiques au sein des parlements peuvent constituer un facteur d'influence substantiel sur les politiques menées, au-delà d'indéniables effets symboliques. Pour établir si de tels effets substantiels existent, et par quelles actions ils sont mis en ceuvre, des analyses plus poussées des activités politiques de ces élus et de leur travail parlementaire devront être réalisées au moyen d'entretiens et de questionnaires. Afin d'obtenir des résultats généralisables, ces analyses devront être comparatives; aussi devront-elles concerner des niveaux politiques divers et tenir compte de contextes politiques différents. C'est pourquoi nous recensons actuellement dans dix Länder les parlementaires issus de l'immigration, afin de leur soumettre ultérieurement le même questionnaire.

On pourra alors savoir s'il existe des différences structurelles entre les élus des différents partis. L'analyse des parlementaires allemands issus de l'immigration que nous avons présentée ci-dessus n'est qu'un premier pas dans cette direction, sa poursuite permettra de développer et de vérifier des hypothèses plus précises sur l'existence de rapports entre la présence de parlementaires issus de l'immigration, les processus parlementaires et les résultats politiques effectifs. 
1. Conférence prononcée au colloque "ÉtrangerFremder in Frankreich und Deutschland", à Strasbourg, les 29 et 30 novembre 2007.

2. Émigrés de l'Union soviétique ou de ses États successeurs, ainsi que de l'Europe de l'Est, d'origine ethnique allemande, en tant que tels titulaires de droit de la nationalité allemande. (N.d.T.)

3. Vogel, Dita, M. Wüst, Andreas, "Paradigmenwechsel ohne Instrumentenwechsel ? Kontinuität und Wandel im Politikfeld Migration”, in : Martin, Seeleib-Kaiser-Antonia, Gohr (Hrsg.), Sozial- und Wirtschaftspolitik unter Rot-Grün, Wiesbaden 2003, p. 265-286 ; Schönwälder, Karen, "Politikwandel in der (bundes)deutschen Migrationspolitik", in : Ulrike, Davy-Albrecht, Weber (Hrsg.), Paradigmenwechsel in Einwanderungsfragen? Überlegungen zum neuen Zuwanderungsgesetz, Baden-Baden 2006, p. 8-22; Castles, Stephen -Davidson, Alastair, Citizenship and Migration. Globalization and the politics of belonging, New York, 2000, p. 93.

4. Hartmut, Esser, Sprache und Integration. Die sozialen Bedingungen und Folgen des Spracherwerbs von Migranten, Frankfurt 2007; Bommes, Michael, "Integration durch Sprache als politisches Konzept", in : Ulrike, Davy-Albrecht, Weber (Hrsg.), Paradigmenwechsel in Einwanderungsfragen? Überlegungen zum neuen Zuwanderungsgesetz, Baden-Baden, 2006, p. 59-86.

5. Miki, C.Kittilson-Katherine, Tate, "Political Parties, Minorities and Elected Office : Comparing Opportunities for Inclusion in the U.S. and Britain", in : Center for the Study of Democracy, Paper 04-06, 2004, Irvine : University of California. http://repositories.cdlib.org/csd/04-06.

6. Vgl. Andreas M. Wüst, "Einstellungen von Parlamentskandidaten gegenüber Einwandererminoritäten in Deutschland und den Niederlanden", Zeitschrift für Parlamentsfragen, 36 (1), 2005, p. 142-152.

7. Milton, M. Gordon, Assimilation in American Life. The Role of Race, Religion and National Origins, New York, 1964, p. 71-81; Hartmut, Esser, Aspekte der Wanderungssoziologie. Assimilation und Integration von Wanderern, ethnischen Gruppen und Minderheiten. Eine handlungstheoretische Analyse, Darmstadt/Neuwied, 1980, p. 221-231; Annette, Treibel, Migration in modernen Gesellschaften. Soziale Folgen von Einwanderung, Gastarbeit und Flucht, Weinheim/München, 1999, p. 141.

8. Phillips, Anne, The Politics of Presence, Oxford, 1995 ; Mansbridge, Jane, "Rethinking Representation", in : American Political Science Review, 97 (4), 2004, p. 515-528.

9. Glenn F. Abney-John D. Hutcheson, "Race, Representation, and Trust : Changes in Attitudes After the Election of a Black Mayor", in : Public Opinion Quarterly, 45 (1), 1981, p. 91-101; Gay, Claudine, "Spirals of Trust? The effect of descriptive representation on the relationship between citizens and their government", in : American Journal of Political Science, 46 (4), 2002, p. 717-733.

10. F. Fenno, Richard, Going Home. Black Representatives and Their Constituents, Chicago 2003, p. 261; Lublin, David, The Paradox Of Representation. Racial Gerrymandering And Minority Interests In Congress, Princeton, 1997, p. $72-97$.

11. Nationaux allemands ou allemands ethniques, expulsés après la Seconde Guerre mondiale de leur territoires de naissance (Heimat) à l'est de l'Allemagne (dans les de frontières de 1914 et de 1937) ainsi que de l'ancienne Autriche-Hongrie. (N.d.T.)

12. À la suite de l'intégration des territoires de l'ex-R.D.A. à l'Allemagne fédérale, l'Union européenne abrite désormais en son sein son premier peuple slave : les Sorabes. (N.d.T.)

13. Rebaptisé "Die Linke"(La Gauche), après sa fusion avec le WASG (Alternative électorale pour le travail et la justice sociale) en 2007. (N.d.T.)

14. Andreas M. Wüst-Dieter Roth, "Parteien, Programme und Wahlverhalten", in : Jens Tenscher (Hrsg.): Wahl-Kampf um Europa, Wiesbaden, 2005, p. 56-85, et p. 65-71.

15. Le système électoral allemand combine les deux. (N.d.T.)

16. Voir aussi M. Messina, Anthony, The Logics and Politics of post-WWII Migration to Western Europe, Cambridge, 2007, p. 194-223.

17. Les pourcentages indiqués sont provisoires, puisque jusqu'à l'heure actuelle nous n'avons pu établir l'origine ethnique que pour cent vingt quatre élus.

18. Cf. Phillips, Anne, The Politics of Presence, Oxford, 1995.

19. Au nombre de quarante environ, les Schrifffïhrer sont élus par le Bundestag en séance plénière, sur proposition des groupes politiques. Assis (deux à la fois) sur des bancs derrière le président de séance, ils assistent celui-ci dans la gestion des séances. (N.d.T.)

20. Cf. "Salto sturzfrei", in : Die Zeit, 8/1995, p. 14.

21. En novembre 2008, Cem Özdemir a été élu co-président des Verts allemands, ce qui sera plus difficile à imiter. (N.d.T.)

22. L'appartenance partisane n'intervient pas à cet égard, et les élus de petits partis ou des partis de gauche ne siègent pas dans plus de commissions que les élus des grands partis ou des partis de droite.

23. Miller, Mark J., Foreign Workers in Western Europe : An Emerging Political Force, New York, 1981.

24. M. Wüst, Andreas, Wie wählen Neubürger? Politische Einstellungen und Wahlverhalten eingebürgerter Personen in Deutschland, Opladen, 2002 ; Vgl. Sara Claro da Fonseca, "Neue Bürger - Neue Kandidaten? Die Parteien im Wettstreit um Migrantenstimmen", in : WZB-Mitteilungen, 114, Berlin, 2006 ; vgl. auch M. Messina, Anthony, op. cit., p. 194-223. 